\title{
A near-infrared survey for Galactic Wolf-Rayet stars
}

\author{
Nicole L. Homeier ${ }^{1}$, Robert D. Blum ${ }^{2}$, Peter S. Conti ${ }^{3}$, Anna Pasquali ${ }^{4}$, \\ and Augusto Damineli ${ }^{5}$ \\ ${ }^{1}$ European Southern Observatory, \\ Karl-Schwarzschild-Straße 2, D-85748 Garching-bei-München, BRD \\ ${ }^{2}$ Cerro Tololo Interamerican Observatory, \\ Casilla 603, La Serena, Chile \\ ${ }^{3}$ Joint Institute for Laboratory Astrophysics, \\ University of Colorado, Boulder, CO 80309-0440, USA \\ ${ }^{4}$ Space Telescope - European Coordinating Facility, ESO, \\ Karl-Schwarzschild-Straße 2, D-85748 Garching-bei-München, BRD \\ ${ }^{5}$ Instituto Astronomico e Geofísico, Universidade de São Paulo, \\ Av. Miguel Stefano 4200, 04301-904 São Paulo, Brasil
}

\begin{abstract}
Most of the Milky Way's evolved massive stellar population is hidden from view. We can attempt to remedy this situation with near-infrared observations, and in this paper we present our method for detecting Wolf-Rayet stars in highly extincted regions and apply it to the inner Galaxy. Using narrow-band filters at $K$-band wavelengths, we demonstrate how WR stars can be detected in regions where they are optically obscured. Candidates are selected for spectroscopic follow-up from our relative line and continuum photometry. The final results of applying this method with a near-IR survey in the Galactic plane will provide a more complete knowledge of the structure of the galactic disk, the role of metallicity in massive stellar evolution, and environments of massive star formation. In this paper we briefly describe the survey set-up and report on recent progress. We have discovered four emission-line objects in the inner Galaxy: two with nebular emission lines, and two new WR stars, both of late WC subtype.
\end{abstract}

\section{Introduction}

Optical surveys within our Galaxy are severely hampered by dust obscuration. Therefore, complete samples must be obtained with longer wavelength observations. Here we describe a survey in the Galactic plane at $K$-band wavelengths, where the extinction is much lower than traditional $V$-band surveys. Our scientific driver is the discovery of the young stellar population in our Galaxy through the detection of evolved massive stars. These stars have strong emission lines, which makes them relatively easy to detect using narrow-band filters.

As a massive O-type star evolves, its spectrum becomes dominated by emission lines, arising either in a dense stellar wind, or in circumstellar material produced by mass loss. Stellar emission lines are most pronounced in Wolf-Rayet stars, which have (O-WR) lifetimes $<10 \mathrm{Myr}$, and thus are excellent tracers of recent star formation, and so also Galactic structure. A complete sample of Galactic WR stars will also enable us to understand the distribution of WN and 
Table 1. New Galactic Center Wolf-Rayet stars.

\begin{tabular}{ccccccc}
\hline \hline object & WR no. & RA (J2000) & Dec (J2000) & $J$ & $H$ & $K$ \\
\hline$\# 1$ & WR 101p & 174542.3 & -285254.7 & & & $\begin{array}{c}10.5: \\
\# 2\end{array}$ \\
WR 102ca & 174613.2 & -284930.1 & 13.3 & 11.0 & $9.4^{b}$ \\
\hline \hline
\end{tabular}

Notes: $a$ : WR numbering system of VIIth Galactic WR Catalogue (van der Hucht 2001); b: from 2MASS.

WC sub-types in a high metallicity environment. Additionally, WR stars are critical components in our quest to understand how star formation proceeds. For example, most of the previously known WRs are relatively isolated, but recent searches in the IR have found a plethora of these objects in stellar clusters near the Galactic Center (e.g., Figer et al. 1999).

\section{Survey description}

We employ four filters situated on prominent lines of He I $2.06 \mu \mathrm{m}$, C IV $2.08 \mu \mathrm{m}$, $\operatorname{Br} \gamma 2.166 \mu \mathrm{m}$, He II $2.19 \mu \mathrm{m}$, and three continuum filters covering wavelengths bluer and redder than each of the line filters $(2.03,2.14$, and $2.248 \mu \mathrm{m}$ ) (Blum \& Damineli 1999; Homeier et al. 2000). In this way we minimize the effects of differential reddening on the continuum of each individual star. Each frame is analyzed with the set of routines collectively called the DоРнот package, a psf-fitting photometry package optimized for large images done in a pipeline process. DOPHOT identifies, classifies, and performs photometry on objects in an image, making successive passes over the image to search each time for fainter objects. Candidates are selected for spectroscopic follow-up based on relative line and continuum photometry and image subtraction with visual examination of the output (Homeier et al. 2003).

In 1996 we began taking data at the $1.5 \mathrm{~m}$ at CTIO, using the Cerro Tololo Infrared Imager (CIRIM). This data comprises approximately 1.5 square degrees of sky near the Galactic Center, half of which we have follow-up spectroscopic observations for. With this data we have discovered two new WR stars and two objects with surrounding nebular emission.

\section{Newly discovered emission-line objects}

Both of the newly discovered WRs appear to be late-type WC stars (WC7-8). Object \#1 is $\sim 8^{\prime}$ from the Quintuplet Cluster, or 20 pc projected distance. We speculate that it has been ejected from the Quintuplet or is a former member of an as-yet undetected massive stellar cluster in the Galactic Center region. Object \#2 is part of the Quintuplet Cluster, and is located just outside the region examined by Figer et al. (1999). Spectra of these two objects are presented in Figure 1, and coordinates are listed in Table 1. Finding charts will be published in an upcoming paper (Homeier et al. 2003).

Of the two objects with nebular emission, one is surrounded by a spherical nebula with strong $\mathrm{H}_{\mathrm{I}}$ lines, including $\mathrm{Br} \gamma 2.166 \mu \mathrm{m}$, and He I $2.06 \mu \mathrm{m}$ emission. This object could be a PN, LBV, or a young O-type star ionizing the surrounding 

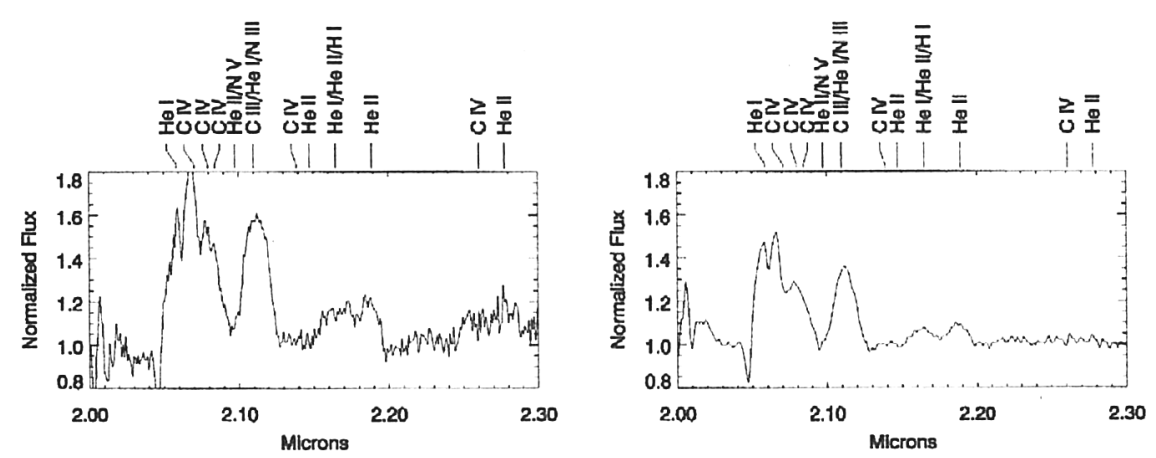

Figure 1. $\quad K$-band spectra of two newly discovered WR stars in our survey. Both spectra are indicative of a late WC type, WC7-8. Left: object \#1, the WR star which does not currently belong to a cluster. Right: object \#2, the WR star discovered in the Quintuplet Cluster.

gas. It is located on the edge of a region with extremely large extinction, and there appears to be more nebular emission a few arcseconds to the south-east. We speculate that this is a star-forming region on the edge of a molecular cloud, and further observations are needed to deduce the distance of this region and whether it contains high-mass stars.

\section{References}

Blum, R.D., Damineli, A. 1999, in: K. A. van der Hucht, G. Koenigsberger \& P.R.J. Eenens (eds.), Wolf-Rayet Phenomena in Massive Stars and Starburst Galaxies, Proc. IAU Symp. No. 193 (San Francisco: ASP), p. 472

Figer, D.F., McLean, I.S., Morris, M. 1999, ApJ 514, 202

Homeier, N.L., Blum, R.D., Conti, P.S., Damineli, A. 2000 AAS, 197.0412

Homeier, N.L., Blum, R.D., Conti, P.S., Damineli, A. 2003, A\&A 397, 585

van der Hucht, K.A. 2001, New Astron. Reviews 45, 135 